\title{
O uso de implantes orbitários de polietileno granulado de ultra-alto peso molecular no reparo de cavidades anoftálmicas
}

\author{
The use of orbital ultra-high molecular weight granulated \\ polyethylene implants in the anophthalmic socket repair
}

João Edward Soranz Filho1, Gustavo Mendes², Regina Kiomi Takahira³, Silvana Artioli Schellini, Cláudia Helena Pellizzon ${ }^{5}$

\section{ReSUMO}

Objetivo: Alterações oculares, em especial a perda de volume nas cavidades evisceradas, promovem uma série de modificaç̃̃es ao paciente tanto funcional do órgão quanto psicológica e estética. Para tanto a procura de um material de baixo custo e com biocompatibilidade tem sido uma constante na literatura. Portanto, esse trabalho teve como objetivo testar experimentalmente implante de polietileno granulado de ultra-alto peso molecular, material de baixo custo, em órbitas de coelhos submetidos à evisceração cirúrgica em vários tempos experimentais, onde foram avaliados aspectos macroscópicos e microscópicose de toxicidade sistêmica do material. Métodos: Para esse estudo foram utilizados coelhos Oryctolaguscuniculus submetidos à evisceração do globo ocular direito e posteriormente implantados com esfera de polietileno granulado de ultra-alto peso molecular e analisados por 15,30,90 e 180 dias pós-implante, com parâmetros macro, microscópios e bioquímicos. Os animais controles foram submetidos ao mesmo procedimento sem, entretanto a colocação do implante. Resultados: Os resultados desse trabalho mostram que o material utilizado no implante de cavidade não apresenta alteração significativa nos parâmetros de peso e bioquímicos quando comparados ao grupo controle. O material implantado apresentou uma grande interação com o tecido do hospedeiro. Conclusão: Os resultados indicam que implante de polietileno granulado de alto peso molecular desenvolvido por uma indústria nacional tem alto potencial para se realizar testes em humanos.

Descritores: Anoftalmia/cirurgia; Olho artificial; Implantes orbitários; Polietilenos/uso terapêutico; Coelhos

\section{ABSTRACT}

Objective: Ocular changes in special due to volume loss in eviscerated cavities promote several psychological aesthetic and functional alterations to patients. For this reason, searches aiming at finding a material of low cost and biocompatibility have been constantly carried out. Test experimentally the implants constituted of ultra-high molecular weight granular polyethylene oflow cost, in orbits of rabbits subjected to surgical evisceration in several experimental times, evaluating the macro and microscope aspects besides the systemic toxicity of the material. Methods:In this study we eviscerated the right ocular globe of rabbits of the species Oryctolaguscuniculus, implanted posteriorly a sphere of granular polyethylene of ultrahigh molecular weight and analysed the effects through macro, micro and biochemical parameters during 15, 30,90 and 180 days after implantation.The control group were submitted the same procedure without the implants. Results: This paper shows that the material used in the cavity does not present significant alteration in the weight and in the biochemistry of the animal; a good assimilation (integration) of the material implanted by the tissue formed was observed inside the implant. Conclusion:The results indicate that ultra-high molecular weight granular polyethylene implant developed by a national industry presents excellent integration in the model used supporting further use in tests with humans.

Keywords:Anophthalmos/surgery; Eye, artificial; Orbital implants; Polyethylenes/therapeutic use; Rabbits

\footnotetext{
1Programa de Pós-graduação Bases Gerais da Cirurgia, Faculdade de Medicina de Botucatu, Universidade Estadual Paulista "Júlio de Mesquita Filho" (UNESP) - Botucatu (SP), Brasil;

2Universidade Estadual Paulista "Júlio de Mesquita Filho" (UNESP) - Botucatu (SP), Brasil;

${ }^{3}$ Universidade Estadual Paulista "Júlio de Mesquita Filho" (UNESP) - Botucatu (SP), Brasil;

${ }^{4}$ Faculdade de Medicina de Botucatu, Universidade Estadual Paulista "Júlio de Mesquita Filho" (UNESP) - Botucatu (SP), Brasil;

${ }^{5}$ Universidade Estadual Paulista "Júlio de Mesquita Filho" (UNESP) - Botucatu (SP), Brasil.
}

Os autores declaram inexistir conflitos de interesse

Recebido para publicação em 13/4/2011 - Aceito para publicação em 15/1/2012 


\section{INTRODUÇÃO}

A perda de um olho pode ser um evento traumático na vida de uma pessoa, resultando em problemas não somente clínicos, mas também emocionais. Pacientes com anoftalmia frequentemente apresentam transtornos psíquicos e ou funcionais que dificultam sua readaptação aos meios social, profissional e familiar ${ }^{(1)}$.

A reconstrução da cavidade anoftálmica passa pela colocação de um implante na órbita, o qual repõe o volume perdido quando da perda do olho ou de seu conteúdo. A prótese externa, em geral em formato de concha e que possui sua superfície externa pintada, de forma a se assemelhar ao olho contralateral.

O material do qual é feito o implante deve ser inerte, isto é, não promover resposta inflamatória, carcinogênica ou alérgica ${ }^{(2,3)}$. Ainda, os implantes necessitam de interação com os tecidos adjacentes para que ocorra melhor mobilidade da prótese externa.

Há diversos materiais sendo utilizados para a confecção dos implantes. Dentre eles, destaca-se o PMMA(polimetilmetacrilato), utilizado desde a década de 40 do século passado e difundido por todo o mundo. Ahidroxiapatita natural foi descrita como material a ser utilizado na confecção dos implantes desde a década de 80 do século XX. Este material já era utilizadopela odontologia e pela ortopedia, em função de sua capacidade de integração ao organismo receptor, quando o implante permite a invasão de tecido fibrovascular altamente vascularizado, diminuindo a possibilidade de extrusão, complicação temida quando se utiliza implantes não integráveis ${ }^{(4)}$. Entretanto, a hidroxiapatita natural é um material proveniente da natureza, de uma fonte esgotável,proveniente de coral, o que justifica o seu alto custo $^{(5)}$.

Implantes confeccionados utilizando hidroxiapatitas sintéticas foram desenvolvidos, com características químicas e funcionais semelhantes à hidroxiapatita natural ${ }^{(6)}$.Entretanto, o carbonato de cálcio presente tanto na hidroxiapatita natural, como na sintética, pode ser fagocitado, levando à redução do volume do implante ${ }^{(7)}$ e metaplasia óssea nos tecidos receptores. ${ }^{(8)}$

Outro material bastante utilizado nos últimos anos tem sido o biovidro. O vidro foi o primeiro material utilizado para confecção de implantes, o que ocorreu no ano de $1900^{(9)}$. Nos últimos anos, modificações na composição química do vidro foram descritas,chegando-se ao biovidro de terceira geração, ou Bioglass ${ }^{\circledR}$, a qual apresentou resultados importantes na regeneração e reparação dos tecidos nos locais onde foi utilizada, devido à ativação dos genes nos tecidos vivos ${ }^{(10)}$.

Por volta do ano de 1990, foi descrito o uso do implante de polietileno poroso (PP), um tipo de polietileno de ultra-alto peso molecular, um material não tóxico e inerte, para reparo da cavidade anoftálmica $^{(11,12)}$.O implante de PP é mais barato que o de hidroxiapatita natural, não causa dano ambiental e a biocompatibilidade e as complicações são comparáveis ao que ocorre quando se utiliza implantes de hidroxiapatita ${ }^{(5)}$.

O implante de PP possui boa mobilidade, pelo fato de que pode ser suturado diretamente aos músculos extraoculares, resultando em melhor transmissão da contração para o implante e propiciando a maior movimentação da prótese externa. Além disso, o implante de PP causa o mínimo de complicações, por ser bem tolerado pelo organismo, com excelente biocompatibilidade, baixa taxa de infecção e de migração celular ${ }^{(5,13)}$.Esse material tem sido utilizado com sucesso como implante no reparo de fratura de órbita e na correção de deformidades faciais estéticas e pós-traumáticas.

O PP fabricado pela Medpor ${ }^{\circledR}$ é bem tolerado pelo organismo e resistente à infecção, promovendo crescimento tecidual no interior do implante, através de poros existentes. Esse material tem características clínicas interessantes, em especial, por aceitar sutura ${ }^{(14)}$. Entretanto, tem custo bastante elevado para se utilizar na rotina hospitalar brasileira, já que carrega com ele o preço da importação.

Assim, o presente estudo foi desenvolvido com o objetivo de avaliar experimentalmente a biocompatibilidade das esferas de polietileno granulado de ultra-alto peso molecular (IPG), desenvolvidas por uma indústria nacional, implantadas em cavidade orbitária eviscerada de coelhos, verificando possíveis alterações celulares e de toxicidade sistêmica.

\section{MÉtodos}

Este é um estudo experimental, prospectivo, com intervenção, realizado na Faculdade de Medicina de Botucatu, Universidade Estadual Paulista "Julio de Mesquita Filho" (UNESP), seguindo todos os Princípios Éticos na Experimentação Animal, adotados pelo Colégio Brasileiro de Experimental (COBEA), tendo sido o protocolo da pesquisa aprovado pela Comissão de Ética na Experimentação Animal (CEEA), protocolo n ${ }^{\circ}$ 77/08 CEEA/Instituto de Biociências da UNESP.

Foram utilizados 48 coelhos da espécie Oryctolaguscuniculus, de ambos os sexos e idade entre três e seis meses, provenientes do Biotério Central da UNESP, Campus de Botucatu. Os animais foram subdivididos aleatoriamente em quatro grupos com 12 animais, sendo que 10 receberam o implante e os outros 2 foram considerados como grupo controle, sendo eviscerados e não recebendo nenhum tipo de implante. Os animais foram operados, sendo sacrificados em diferentes tempos experimentais, a saber: $15,30,90$ e 180 
dias após a cirurgia de evisceração, com a colocação do implante na cavidade orbitária.

Foram utilizados implantes esféricos, confeccionados em polietileno granulado de ultra-alto peso molecular (IPG) pela Cloud Tecnologia Informação Ltda. em Sorocaba (SP). Os implantes foram confeccionados utilizando polietileno no estado de pó,com partículas de média densidade, de ultra-alto peso molecular ( $\left.2 \times 10^{6} \mathrm{~g} / \mathrm{mol}\right)$, classificado como não tóxico e inerte, com ponto de fusão entre 125 a $135^{\circ} \mathrm{C}$, com densidade de $0,920-0,935 \mathrm{~g} / \mathrm{cm}^{3}$ e não solúvel. A caracterização físicoquímica do material empregado no implante foi feita pela Cloud Tecnologia Informação Ltda., firma que forneceu o material para a pesquisa, sem nenhum custo e qualquer benefício aos pesquisadores desse artigo.

\section{Procedimento experimental}

Todos os procedimentos cirúrgicos foram realizados no Laboratório de Cirurgia Experimental do Departamento de Oftalmologia, Otorrinolaringologia e Cirurgia de Cabeça e Pescoço, da Faculdade de Medicina de Botucatu UNESP. Os procedimentos foram realizados sob condições de assepsia, sempre pelo mesmo cirurgião. Os animais após prévia anestesia, utilizando Zoletil $®$ (cloridrato de tiletamia e zolazepanVirbac, Brasil) na dose de $15 \mathrm{mg} / \mathrm{kg}$, associado com Xilazina(cloridrato de xilazinaVirbac, Brasil) na dose de $0,015 \mathrm{mg} / \mathrm{kg}$, foram submetidos à evisceração do olho. Onde a conjuntiva bulbar superior foi aberta utilizando-se tesoura de argola, e isolando o músculo reto superior para abertura da esclera com bisturi lâmina 15 sob o local de sua inserção.Após esse procedimento, o conteúdo do olho direito foi eviscerado utilizando colher de evisceração,com substituição do conteúdo removido pelo implante de $10 \mathrm{~mm}$ de diâmetro, o qual foi suturado contínuo da esclera com pontos de Mersilene 6-0. Nos animais do grupo controle não ocorreu a colocação do implante. Após a cirurgia os animais receberam duas gotas de colírio antibiótico Maxiflox D (Cloridrato de Ciprofloxacino e Dexametasona, Latinofarma, Brasil).

\section{Avaliação de toxicidade sistêmica}

No início do experimento (P0) todos os animais foram submetidos à pesagem, análise clínica geral e posteriormente foi retirado sangue periférico da veia auricular,usando seringa descartável de $5 \mathrm{ml}$ com agulha hipodérmica de $0,45 \times 13 \mathrm{~mm}$, para análise bioquímica, a fim de avaliar possível toxicidade sistêmica que fosse induzida pelo implante. Nova coleta do sangue periférico foi realizada imediatamente antes do sacrifício dos animais, que ocorreu 15 (P1), 30 (P2), 90 (P3) e 180 (P4) dias após a colocação do implante na cavidade eviscerada.

\section{Avaliação clínica}

A avaliação do peso dos animais foi feita anteriormente à cirurgia e semanalmente até o sacrifício, den- tro dos tempos experimentais. Diariamente a saúde geral com base no comportamento dos animais, sendo avaliados padrão de pelagem e ganho de peso, além da verificação da área cirúrgica .

\section{Exames bioquímicos}

As amostras de sangue foram centrifugadas para obtenção do soro e processadas no Laboratório Clínico Veterinário da Faculdade de Medicina Veterinária e Zootecnia da UNESP de Botucatu/SP,Departamento de Clínica Veterinária, para a determinação das atividades séricas de AspartatoAminotransferase (AST), Alanina Aminotransferase (ALT), Fosfatase Alcalina (FA) e Gama-glutamiltransferase (GGT) para avaliação da função hepática. As concentrações séricas de ureia e creatinina foram determinadas para avaliação da função renal. As análises foram realizadas em equipamento de bioquímica automatizada Cobas Mira Plus ${ }^{\circledR}$ (Roche Diagnostic, Basel, Switzerland) e reagentes comerciais Katak ${ }^{\circledR}$ (Katal Biotecnológica Indústria e Comércio Ltda., Belo Horizonte, Brasil).

\section{Retirada do implante para análise}

Transcorrido o período experimental e após a coleta de sangue, nos momentos P1, P2, P3 e P4, os animais foram sacrificados utilizando dose excessiva de Xilazina(cloridrato de xilazinaVirbac, Brasil). Imediatamente após a morte do animal, os conteúdos das órbitas direitas foram enucleados e colocados em formol tamponado $10 \%$ durante 24 horas a $4^{\circ} \mathrm{C}$ para fixação. Em seguida, foram processados para inclusão em Paraplast ${ }^{\circledR}$ X-TRA (Ted Pella INC, EUA).

\section{Análise histológica}

Após a inclusão, as amostras foram cortadas com espessura de $5 \mu \mathrm{m}$ e colocadas sobre lâminas histológicas, coradas por Hematoxilina e Eosina ${ }^{(15)}$.A avaliação das lâminas foi feita em microscópio DMLB Leica (Alemanha), acoplado ao software 3.1 Q-WinLEICA (Inglaterra), avaliando-se a presença de células, interação do tecido adjacente ao implante e, quando presente, a resposta tecidual inflamatória.

\section{Análise morfométrica}

Foi realizada a quantificação de células totais, utilizando cinco campos de cada animal, fotomicrografados no Sistema de Fotomicrografia microscópio DMLB Leica(Alemanha), acoplado ao programa 3.1 Q-Win Leica (Inglaterra). Posteriormente, as imagens foram analisadas utilizando o software AVSoftBioview 4 Seeker Module (AVSoft, Brasil).

\section{Análise estatística}

Opeso dos animais foi analisado pelo teste de WilcoxonRank Sum Test, utilizando o programa R (ver- 
são 2.11.1, 31/05/2010). Para as análises bioquímicas e morfométricas, foi utilizado o teste não paramétrico $t$ Student, para a comparação das médias de duas amostras independentes e aleatórias ${ }^{(16)}$.

\section{Resultados}

Os animais submetidos à evisceração da cavidade orbitária e que receberam o implante de IPG apresentaram bom desenvolvimento clínico, ausência de secreção ou qualquer alteração significativa na região da órbita direita. O implante promoveu adequada reposição do volume orbital durante todo o período experimental (Figura 1).

Na Tabela 1, observa-se as medidas dos pesos nos momento P0 até o sacrifício do momento experimental do grupo, sendo possível observar que todos os animais apresentaram o mesmo padrão de ganho de peso, não havendo interferência da presença do implante neste parâmetro.

Os resultados das análises bioquímicas para uréia, creatinina, ALT, AST, FA e GGT podem ser observadas na Tabela 2; não ocorreram alterações importantes entre os grupos controle e implantados, juntamente com o dado anterior do peso, o que reforça a hipótese de não haver interferência do material implantado nos animais.

Quando analisada a interação do implante com o tecido biológico do animal, observa-se interação bastante satisfatória, não ocorrendo processos inflamatórios nem alterações da esclera ao redor da cavidade.A resposta dos tecidos com hospedeiro na colocação do implante e a interação do tecido neoformado infiltrado ao redor do implante, pode-se notar que há presença desse tecido com matriz extracelular acidófila e células com morfologia similar a fibroblasto e também a presença de

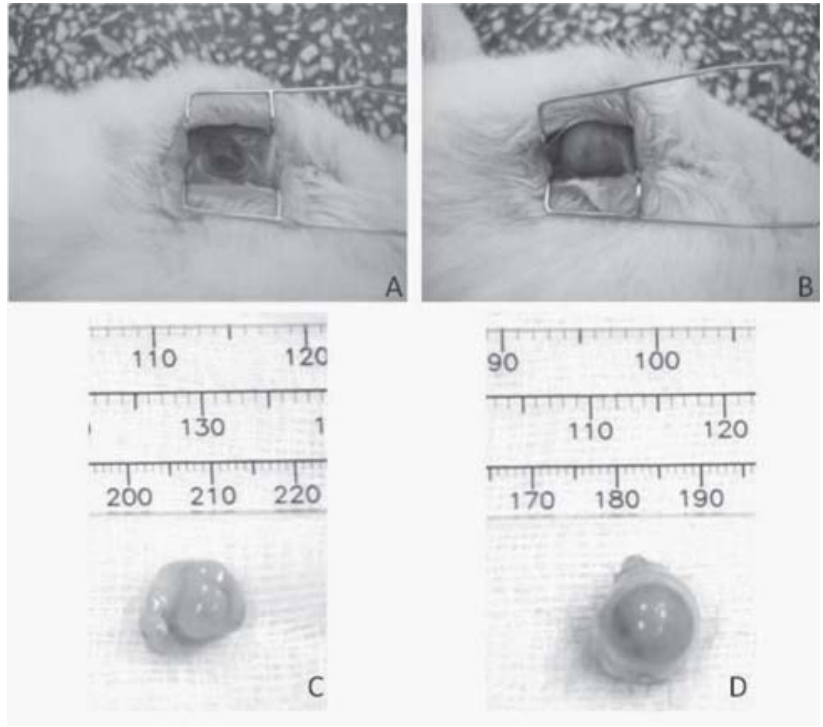

Figura 1: Olhos dos coelhos submetidos ao implante poroso após 180 dias da implantação; em A observa-se o olho do animal controle, sem a reparação do volume da cavidade após a enucleação, onde pode se notar uma alteração de volume da cavidade bastante significativa em $\mathrm{C}$, quando comparada com a do animal submetido ao implante em B e D; note que o animal submetido ao implante em B tem um padrão de volume orbital mantido

vasos sanguíneos com hemácias bastante evidentes (Figura 2).Quando analisada a esclera nota-se a constituição de tecido conjuntivo denso apresentando-se altamente acidófilo com fibras dispostas paralelamente, com a presença principalmente de fibroblasto. Já analisando a quantidade total de células entre os dois grupos estudados, não houve alteração nesse número, forte indicativo de que o material implantado não promove qualquer tipo de alteração inflamatória local (Tabela 3).

Tabela 1

\begin{abstract}
Peso médio dos animais submetidos à evisceração e implantados com prótese de polietileno granular de ultra-alto peso molecular, durante os diferentes períodos experimentais nos momentos inicial e final; dados expressos em média \pm desvio padrão
\end{abstract}

\begin{tabular}{|c|c|c|c|c|c|c|c|c|}
\hline & \multicolumn{2}{|c|}{ Inicial } & \multicolumn{2}{|c|}{ Inicial } & \multicolumn{2}{|c|}{ Inicial } & \multicolumn{2}{|c|}{ Inicial } \\
\hline & $\begin{array}{l}\text { Grupo } \\
15 \text { dias }\end{array}$ & Controle & $\begin{array}{l}\text { Grupo } \\
30 \text { dias }\end{array}$ & Controle & $\begin{array}{l}\text { Grupo } \\
90 \text { dias }\end{array}$ & Controle & $\begin{array}{c}\text { Grupo } \\
180 \text { dias }\end{array}$ & Controle \\
\hline Peso(Kg) & 2,0 & 2,2 & 1,2 & 1,2 & 1,3 & 1,3 & 1,3 & 1,4 \\
\hline \multirow[t]{3}{*}{ DP } & 0,2 & 0,2 & 0,1 & 0,3 & 0,1 & 0,1 & 0,2 & 0,1 \\
\hline & \multicolumn{2}{|c|}{ Final } & \multicolumn{2}{|c|}{ Final } & \multicolumn{2}{|c|}{ Final } & \multicolumn{2}{|c|}{ Final } \\
\hline & $\begin{array}{l}\text { Grupo } \\
15 \text { dias }\end{array}$ & Controle & $\begin{array}{l}\text { Grupo } \\
\mathbf{3 0} \text { dias }\end{array}$ & Controle & $\begin{array}{l}\text { Grupo } \\
90 \text { dias }\end{array}$ & Controle & $\begin{array}{c}\text { Grupo } \\
180 \text { dias }\end{array}$ & Controle \\
\hline Peso(Kg) & 2,7 & 2,7 & 2,7 & 2,6 & 4,2 & 4,0 & 4,2 & 3,9 \\
\hline DP & 0,3 & 0,1 & 0,2 & 0,4 & 0,2 & 0,4 & 0,2 & 0,1 \\
\hline
\end{tabular}




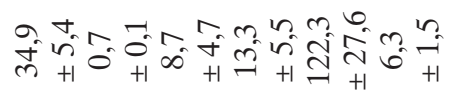

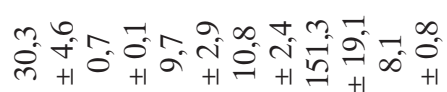

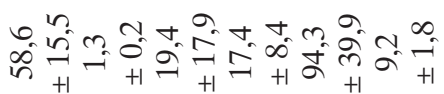

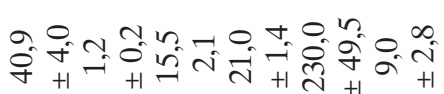

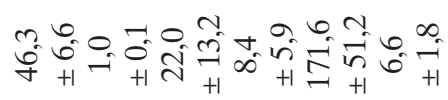

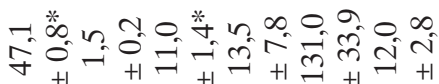

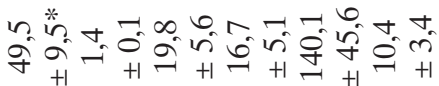

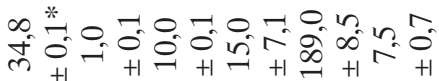

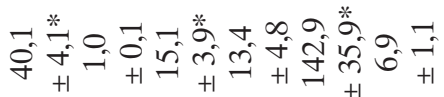

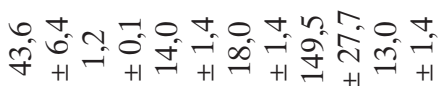

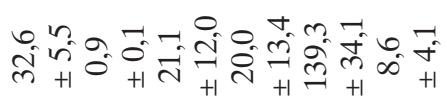

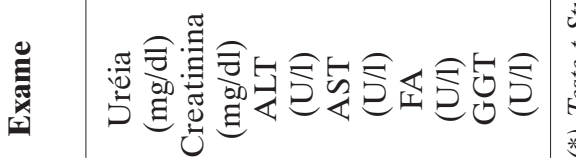

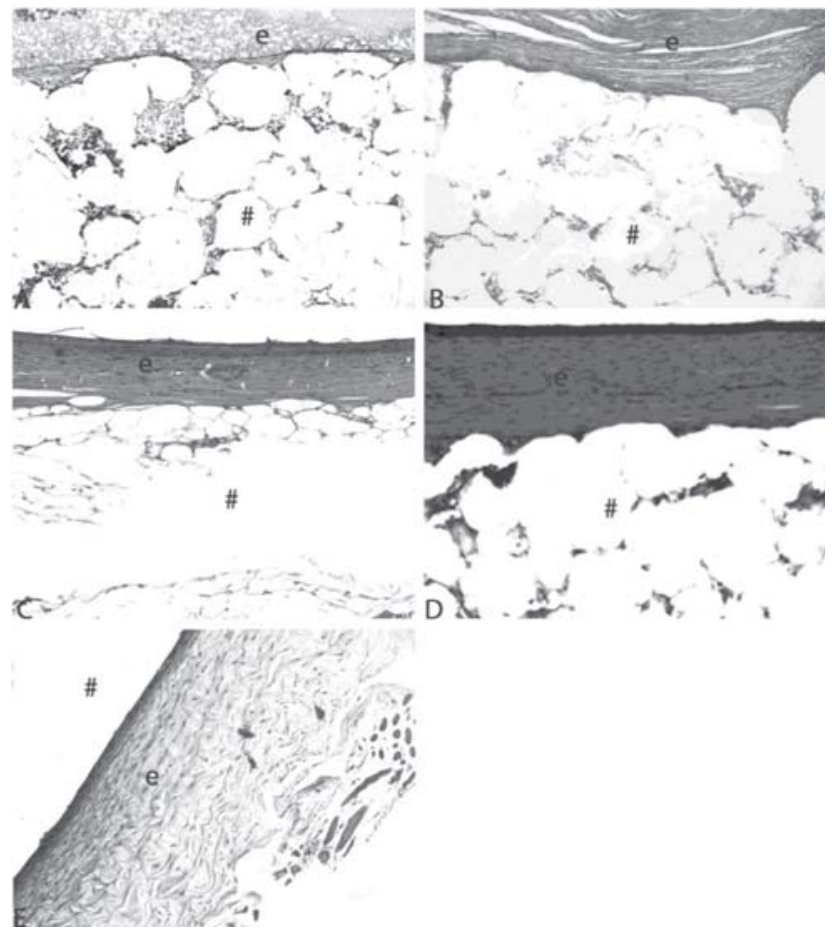

Figura 2: Fotomicrografia dos olhos de coelhos submetidos à evisceração; em A, C, E e G são dos olhos do grupo controle que foram eviscerados mas não submetidos à reposição da cavidade orbital; B, D, F e H observa-se o material dos olhos do grupo implantado; (*) é a região da esclera e \# é a região da cavidade eviscerada; note que os grupos implantados apresentam a presença de infiltração tissular (seta), sendo que A e B são do grupo com 15 dias após o implante, já C e D com 30 dias; E e F com 90 dias e $\mathrm{G}$ e $\mathrm{H}$ com 180 dias; Barra $100 \mu \mathrm{m}$

\section{Discussão}

O estudo de novos materiais para reconstrução da cavidade anoftálmica tem como objetivo a redução da resposta biológica do organismo ao material implantado, com integração tecidual do implante ao hospedeiro. $\mathrm{Na}$ década de 80 do século XX, iniciou-se o desenvolvimento da chamada segunda geração de biomateriais com foco em materiais bioativos, os quais promovem ação controlada ao meio biológico com reação positiva ou inerte ao ambiente fisiológico da região implantada ${ }^{(17)}$.

Este estudo foi desenvolvido para verificação da interação biológica ao implante de polietileno de ultraalto peso molecular (IPG), desenvolvido por uma indústria nacional, para reposição de volume da cavidade orbitária, como alternativa ao uso de próteses importadas.

Para tanto, o material foi analisado do ponto de vista da indução de toxicidade e também de aceitação pelos tecidos orbitários, analisando-se a intensidade, tipo e distribuição de reação tecidual induzida, em diferentes tempos experimentais, procurando-se conhecer a re- 


\section{Tabela 3}

\section{Contagem de células totais em diferentes tempos experimentais após o implante de polietileno com dados expressos em média e \pm desvio padrão}

\begin{tabular}{crr}
\multicolumn{3}{c}{ Número de células totais } \\
\hline Grupo & \multicolumn{1}{c}{ Controle } & \multicolumn{1}{c}{ Implantado } \\
\hline 15 dias & $1336,00 \pm 94,48$ & $1250,26 \pm 299,96$ \\
30 dias & $828,00 \pm 310,42$ & $975,94 \pm 285,35$ \\
90 dias & $9,17 \pm 71,42$ & $799,23 \pm 255,36$ \\
180 dias & $1267,80 \pm 513,59$ & $670,98 \pm 241,74$
\end{tabular}

(*) Teste $t$-Student

ação aguda e a longo prazo.

Coelhos são modelos experimentais adequados para este tipo de estudo, pois apresentam rápido crescimento e manejo relativamente fácili(18), para tanto foram desenvolvidos implantes IPG com tamanho de $10 \mathrm{~mm}$, considerado ideal para o tamanho da cavidade córneoescleral do animal, pois nesse estudo optou-se pela manutenção da córnea do animal.

Apesar de as esferas de IPG possibilitarem a sutura direta do implante à musculatura extraocular, fator importante para promover motilidade da prótese ${ }^{(19,20)}$, no presente estudo a esfera foi colocada na capa córneoescleral, já que se optou pela evisceração e não pela enucleação.

A análise clínica dos indivíduos experimentais foi realizada diariamente, não tendo sido observado secreção, sangramento, extrusão ou deslocamento dos implantes orbitários. Os pesos corporais foram aferidos semanalmente, que indicaram o desenvolvimento normal, com ganho de peso tanto nos grupos implantados como no de controle. Essa observação clínica aponta que o material utilizado na confecção do implante não promoveu alteração no desenvolvimento do animal.

As análises do sangue retiradas no início do experimento e comparadas com o mesmo animal no final do tempo experimental (amostras dependentes), para creatinina, uréia, ALT, AST, FA e GGT, mostraram que não houve alterações significativas entre os grupos controle e implantado. Dados semelhantes aos obtidos nesse trabalho foram também observados quando se utilizou implantes de biovidro e biovidrocerâmico ${ }^{(21)}$ em cavidades anoftálmicas de coelhos. Ainda, comparando-se os resultados de exames bioquímicos com os de coelhos normais, os níveis foram semelhantes, indicando que o IPG não induz resposta sistêmica prejudicial aos animais.

Os resultados na análise morfológica da interação do implante com a cavidade orbital mostraram que há invasão tecidual nos poros do implante, com matriz extracelular acidófila com vasos sanguíneos associados com células típicas, em especial fibroblastos. Nosso estudo mostrou que não ocorreu qualquer processo inflamatório, sem presença em especial de neutrófilos, macrófagos ou linfócitos típicos, em especial na esclera e tecido adjacente, essa observação ocorreu em todos os tempos experimentais utilizando o IPG. Podemos afirmar ainda com base nos resultados que o IPG tem um grande potencial para induzir a neoformação tecidual, em função do tecido neoformado apresentar vascularização em seu interior e ausência de evidência de processo inflamatório.

Esses dados diferem de outros estudos feitos pelo mesmo grupo de pesquisadores que desenvolveram o presente estudo,quando foram avaliados coelhos com cavidade anoftálmica que receberam implantes debiovidro e biovidrocerâmico, que apresentaram edema e elevada infiltração de neutrófilos no início do período de experimentação ${ }^{(21)}$ ou em ratos que receberam implantes de quitosana no subcutâneo, os quais apresentaram elevada resposta inflamatória nos primeiros 15 dias após o implante, notadamente com presença de linfócitos e neutrófilos ${ }^{(22)}$.

O IGP apresentou comportamento semelhante ao Medpor ${ }^{\circledR}$ implantado em um período de 16 meses pela técnica de evisceração ${ }^{(23)}$, isto é, uma fina camada tecidual de tecido fibrovascular de densidade variável interagindo com o implante e raras células inflamatórias.

A preocupação com o processo inflamatório e em especial com a extrusão da prótese implantada é bastante presente na literatura ${ }^{(24)}$, sendo um fator importante no desenvolvimento de novo material para se utilizar em implantes. Nesse sentido, o IPG mostrou-se promissor, uma vez que não apresentou alteração celular entre os animais dos grupos controle e implantado e nenhum caso de extrusão ou migração do implante.

Quando se comparam esferas de hidroxiapatita natural e Medpor ${ }^{\circledR}$ (polietileno), a resposta inflamatória em cavidades de coelhos foi maior nos animais implantados com hidroxiapatita natural, indicando que o polietileno tem uma reação mais simbiótica com o tecido biológico ${ }^{(25)}$. Com implante de hidroxiapatita sintética em cavidade anoftálmica em ratos, observou-se a presença de células fagocíticas, como células gigantes de corpo estranho ${ }^{(26)}$. Neste trabalho, analisando de maneira qualitativa, o aspecto morfológico da interação implante-cavidade orbital não apresentou qualquer tipo de alteração que permitisse a identificação de processo inflamatório. Esse resultado foi corroborado com a contagem de células totais da região de interface, que não apresentou qualquer alteração celular entre os grupos utilizados nesse estudo, uma vez que o processo inflamatório promove alteração no número total de células, podendo ser altamente lesivo ao paciente, em função de aumento específico de células polimorfonuclerares $^{(27)}$. 


\section{CONClUSÃO}

Frente aos resultados obtidos nesse estudo, os autores concluem que o IPG pode ser uma nova opção de material para reparação da cavidade anoftálmica, em função de não induzir toxicidade, não provocar reação inflamatória exacerbada e evoluir ao longo prazo com a manutenção do volume implantado no modelo utilizado nesse trabalho.

Agradecimentos: Suporte Financeiro: FAPESP 09/52298-8; Bolsa Iniciação Científica para o autor Mendes, G.

\section{ReferÊNCIAS}

1. Cabral LGM, Martelli Júnior H, Leite DM, Sabatini Júnior D, Freitas ABDA, Miranda RT, et al. Perfil biopsicossocial de portadores de anoftalmia no sul de Minas Gerais - Brasil. Arq Bras Oftalmol. 2008;71(6):855-9.

2. Lee S, Maronian N, Most SP, Whipple ME, McCulloch TM, Stanley RB, Farweel DG. Porous high-density polyethylene for orbital reconstruction. Arch Otolaryngol Head Neck Surg. 2005;131(5):446-50.

3. Frodel JL, Lee S. The use ofhigh-density polyethylene implants in facial deformities. Arch Otolaryngol Head Neck Surg. 1998;124(11):1219-23.

4. Perry AC. Integrated orbital implants.Adv Ophthalmic PlastReconstrSurg. 1990;8:75-81.Review.

5. Goiato MC, Haddad MF, dos Santos DM, Pesqueira AA, Ribeiro Pdo P, Moreno A. Orbital implantsinsertionto improve ocular prosthesesmotility. J CraniofacSurg. 2010;21(3):870-5.

6. Costantino PD, Friedman CD, Jones K, Chow LC, Sisson GA. Experimental hydroxyapatite cement cranioplasty. PlastReconstr Surg. 1992;90(2):174-85; discussion 186-91.

7. Schellini SA, Marques ME, Ranzanin JJ, Taga EM. Synthetic hydroxyapatite in anophthalmic socket reconstruction. Invest Ophthalmol Vis Sci. 1998;39:502.

8. Schellini SA, Hoyama E, Padovani CR, Ferreira VL, Roça R. Complicações com usode esferas não integráveis e integráveis na recontrução da cavidade anoftálmica. ArqBras Oftalmol. 2000;63(3):175-8.

9. den Tonkelaar I, Henkes HE, van Leersum GK. Herman Snellen (1834-1908) and Müller's'reform-auge'. A short history of the artificial eye. Doc Ophthalmol.1991;77(4):349-54.

10. Hench LL. The story of Bioglass. J Mater Sci Mater Med. 2006;17(11):967-78.

11. Rubin LR. Polyethylene as a bone and cartilage substitute: a 32-year retrospective experience. In: Rubin LR. Biomaterial reconstructive surgery. St. Louis: Mosby; 1983. p. 474-93.

12. Warren NP.A short history of total hip replacement. In: Coombs R, Gristina A, Hungerford DS. Joint replacement: state of art. St. Louis: Mosby; 1990.

13. Chen YH, Cui HG. High density porous polyethylene material (Medpor) as an unwrapped orbital implant.J ZhejiangUniv Sci B. 2006;7(8):679-82.
14. Karesh JW, Dresner SC. High-density porous polyethylene (Medpor) as a successful anaphthalmic socket implant. Ophthalmology. 1994;101(10):1688-95; discussion 1695-6.

15. BehmerOA, Tolosa EMC, Freitas Neto AG. Manual de técnicas para histologia normal e patológica. São Paulo: Edart; 1976. 241 p.

16. Corder GW, Foreman DI. Nonparametric statistics for non-statisticians: a step-by-step approach. New Jersey: Wiley; 2009. 247p.

17. Hench LL, Thompson I. Twenty-first century challenger for biomaterials. J R Soc Interface. 2010;7Suppl 4:S379-91.

18. Shiratori CA, Schellini SA, Yamashita S, Padovani CR, Rossa R. Avaliação das dimensões orbitárias de coelhos após enucleação e evisceração Arq Bras Oftalmol. 2005;68(2):235-9.

19. Chalasani R, Poole-Warren L, Conway RM, Ben-Nissan B. Porous orbital implants in enucleation: asystematic review. Surv Ophthalmol. 2007;52(2):145-55.

20. Sadiq SA, Mengher LS, Lowry J, Downes R. Integrated orbital implants-a comparison of hydroxyapatite and porous polyethylene implants. Orbit. 2008;27(1):37-40.

21. Brandão SM. Análise dabiocompatibilidade de cones de biovidro e biovitrocerâmico em cavidade eviscerada de coelho [dissertação]. Botucatu: Faculdade de MedicinadaUniversidade Estadual Paulista "Julio de Mesquita Filho"; 2010.

22. Brito MKM, Schellini SA, Padovani CR,Pellizzon CH, Trindade Neto CG.Inclusões de quitosana no subcutâneo de rato: avaliação clínica, histológica e morfométrica. AnBrasDermatol. 2009;84(1):35-40.

23. Núñez Sánchez A, Reche Sainz JA, Sanz López A, Mateos Sánchez E, García Llanes G, San Miguel Fraile P, Fernández Escámez CS. Integracíon de los implantes orbitarios de polietileno (Medpor). Estudio histológico. Arch Soc Esp Oftalmol. 2001;76(1):25-9.

24. Custer PL, TrinkausKM. Porous implant exposure: incidence, management and morbidity.Ophthal Plast Reconstr Surg. 2007;23(1):1-7.Review.

25. Goldberg RA, Dresner SC, Braslow RA, Kossovsky N, Legmann A. Animal model of porous polyethylene orbital implants.OphthalPlast Reconstr Surg. 1994;10(2):104-9.

26. Ranzani JJ, Rahal SC, Schellini SA, Marques ME, Taga EM. Repair of the anophthalmic cavity of rats with synthetic hydroxyapatite. Braz J MedBiol Res. 1997; 30(10):1181-6.

27. Paulo NM, Lima FG, Siqueira Júnior JT, Fleury LFF, Sant'Ana FJF, Borges AC, Telles TC. Membrana de látex da seringueira (Hevea brasiliensis), com e sem polilisina a 0,1por cento e tela de marlex na reconstrução de defeitos iatrogênicos da parede abdominal de ratos. Acta Cir Bras. 2005;20(4):305-10.

\section{Endereço para correspondência:}

Cláudia Helena Pellizzon

Departamento de Morfologia Instituto de Biociências da UNESP

Caixa Postal 510

CEP 18618-970 - Botucatu (SP), Brasil

Tel/fax: 55(14) 3811-6264

E-mail: claudia@ibb.unesp.br 